\title{
Direct hemoperfusion with polymyxin B-immobilized fiber column in a patient with acute exacerbation of idiopathic pulmonary fibrosis
}

\author{
Shin Young Kim², Jin Han Park², Hyo Jung Kim², Hang Jea Jang², Hyun Kuk Kim², Seung Hoon Kim, \\ Jae Ha Lee ${ }^{2}$
}

${ }^{1}$ Department of Internal Medicine, St. Vincent's Hospital, College of Medicine, The Catholic University of Korea, Suwon; ${ }^{2}$ Division of Pulmonology and Critical Care Medicine, Department of Internal Medicine, Inje University Haeundae Paik Hospital, Inje University College of Medicine, Busan, Korea

Idiopathic pulmonary fibrosis (IPF) is a progressive fibrosing interstitial lung disease characterized by dyspnea and a worsening of the lung function. Acute exacerbations of idiopathic pulmonary fibrosis (AE-IPF) are defined by a clinically significant respiratory deterioration, that typically develops in less than 1 month, accompanied by new radiologic abnormalities on high-resolution computed tomography, including diffused and bilateral ground-glass opacification, along with an absence of other obvious clinical etiologies. Recently, AE-IPF has gained significant importance as a major cause of mortality and morbidity. However, despite the extremely poor prognosis of the condition, no well-validated therapeutic interventions are currently available. Therefore, novel treatment modalities are being investigated and applied in addition to conventional treatments. Among them, several studies have reported that a direct hemoperfusion with a polymyxin B-immobilized fiber column (PMX-DHP), developed for endotoxin removal in septic shock, has an effect on AE-IPF. We describe two cases of PMX-DHP treatment with conflicting results. One patient successfully recovered via a PMXDHP in severe AE-IPF that required extracorporeal membrane oxygenation (ECMO). PMX-DHP subsequently improved oxygenation $\left(\mathrm{PaO}_{2} / \mathrm{FiO}_{2}\right.$ ratio) and decreased the levels of inflammatory markers (interleukin-6, C-reactive protein, and white blood cells). The patient dramatically recovered without the need for ECMO. PMX-DHP may be considered an alternative therapy in $\mathrm{AE}-\mathrm{IPF}$ patients requiring mechanical ventilation or ECMO.

Key Words: idiopathic pulmonary fibrosis; polymyxin B; prognosis

The prognosis for idiopathic pulmonary fibrosis (IPF) patients with acute exacerbation of idiopathic pulmonary fibrosis (AE-IPF) is devastating. Currently, no well-validated effective treatment modalities are available and the mean survival time from the onset of acute exacerbation is only 1.5 months [1]. The prognosis is very poor, particularly in cases of severe hypoxemia that require intensive care unit care or mechanical ventilation. Direct hemoperfusion with a polymyxin B-immobilized fiber column (PMX-DHP) which was developed for endotoxin removal is currently being considered for use in sepsis as an alternative therapy. Moreover in case reports, this approach was beneficial in patients with Gram-positive bacterial infections and endotoxin-negative infections [2]. Recently, a study reported that PMX-

\section{Case Report}

Received: January 22, 2020

Revised: March 6, 2020

Accepted: March 7, 2020

Corresponding author

Jae Ha Lee

Division of Pulmonology and Critical

Care Medicine, Department of

Internal Medicine, Inje University

Haeundae Paik Hospital, Inje

University College of Medicine, 875

Haeun-daero, Haeundae-gu, Busan

48108, Korea

Tel: +82-51-797-0457

Fax: +82-51-797-2210

E-mail: anilleus@naver.com

Copyright (@) 2020 The Korean Society of Critical Care Medicine

This is an Open Access article distributed under the terms of Creative Attributions Non-Commercial License (https:// creativecommons.org/li-censes/by-nc/4.0] which permits unrestricted noncommercial use, distribution, and reproduction in any medium, provided the original work is properly cited. 
DHP was effective in patients with acute respiratory distress syndrome (ARDS) and AE-IPF [3-5]. Herein, we describe the outcomes of applying PMX-DHP in two patients with severe AE-IPF.

\section{CASE REPORTS}

\section{Case 1}

A 63-year-old man presented in the emergency room with dyspnea and cough. He had been admitted to a local medical clinic 3 weeks prior due to mild shortness of breath, and chest computed tomography (CT) showed a definite usual interstitial pneumonia pattern, based on the 2015 American Thoracic Society (ATS)/European Respiratory Society (ERS) guidelines [6]. Compared to 3 weeks ago, the chest X-ray showed significantly increased infiltration on both lung fields (Figure 1). The patient was diagnosed with hypertension 5 years ago, has been taking anti-hypertensives, and was previously a smoker. The pulmonary function test results indicated that the forced expiratory volume in 1 second/forced vital capacity (FEV1/FVC) ratio was $115 \%$, the predicted FVC was $41 \%$ and the predicted diffusing capacity of the lung for carbon monoxide (DLco) was $37 \%$. According to the sex-age-lung physiology variables (GAP) index, the severity of IPF was estimated as stage 2 [7]. The vital signs at the time of visit were: blood pressure, 205/114 mm Hg; pulse rate, 114/min; respiratory rate, 31 breaths/min; body temperature $36.8^{\circ} \mathrm{C}$; and an oxygen saturation of $86 \%$ at room air. Blood tests performed at the time of admission revealed leukocytes, 21,000/ $\mu \mathrm{l}$ (neutrophils, $81.2 \%$ ); C-reactive protein (CRP), $20.76 \mathrm{mg} / \mathrm{dl}$; procalcitonin, $0.11 \mathrm{ng} / \mathrm{ml}$; brain natriuretic peptide, $135.5 \mathrm{pg} / \mathrm{ml}$; lactate dehydrogenase, $545 \mathrm{IU} / \mathrm{L}$; creatine kinase, $60 \mathrm{U} / \mathrm{L}$; and interleukin-6 (IL-6), 68.5 pg/ml. Arterial blood gas analysis revealed a $\mathrm{pH}$ of $7.42 ; \mathrm{pO}_{2}, 43 \mathrm{~mm} \mathrm{Hg}$; $\mathrm{pCO}_{2}, 34 \mathrm{~mm} \mathrm{Hg}$; and lactate, $2.0 \mathrm{mmol} / \mathrm{L}$. Chest CT revealed a bilateral subpleural reticular shadow and honey-combing with an increased extent of ground-glass opacity (Figure 2A). With no other risk factors associated with interstitial lung disease, the patient was diagnosed with AE-IPF, according to the 2016 ATS/ERS criteria. The patient's partial pressure of oxygen was $43 \mathrm{~mm} \mathrm{Hg}$ with no improvements in hypoxemia following the application of a high-flow nasal cannula, at a setting of $\mathrm{FiO}_{2}$ 1.0, and flow $60 \mathrm{~L} / \mathrm{min}$. We initiated mechanical ventilation and treatment with corticosteroid pulse therapy (methylprednisolone $1 \mathrm{~g}$ /day for 3 days), and antibiotic therapy. ECMO was required because the $\mathrm{PaO}_{2} / \mathrm{FiO}_{2}$ ratio was continuously $<50$, despite the maximal support provided via mechanical ventilation (setting $\mathrm{FiO}_{2} 1.0$ and positive end expiratory pressure level over 15) with use of a neuromuscular blocker, and for bridging therapy for future lung transplantation. Therefore, we decided to start PMX-DHP prior to ECMO to try and improve the patient's condition. After 14 hours of hospitalization, we performed two sessions of PMX-DHP (Toraymyxin 20-R;
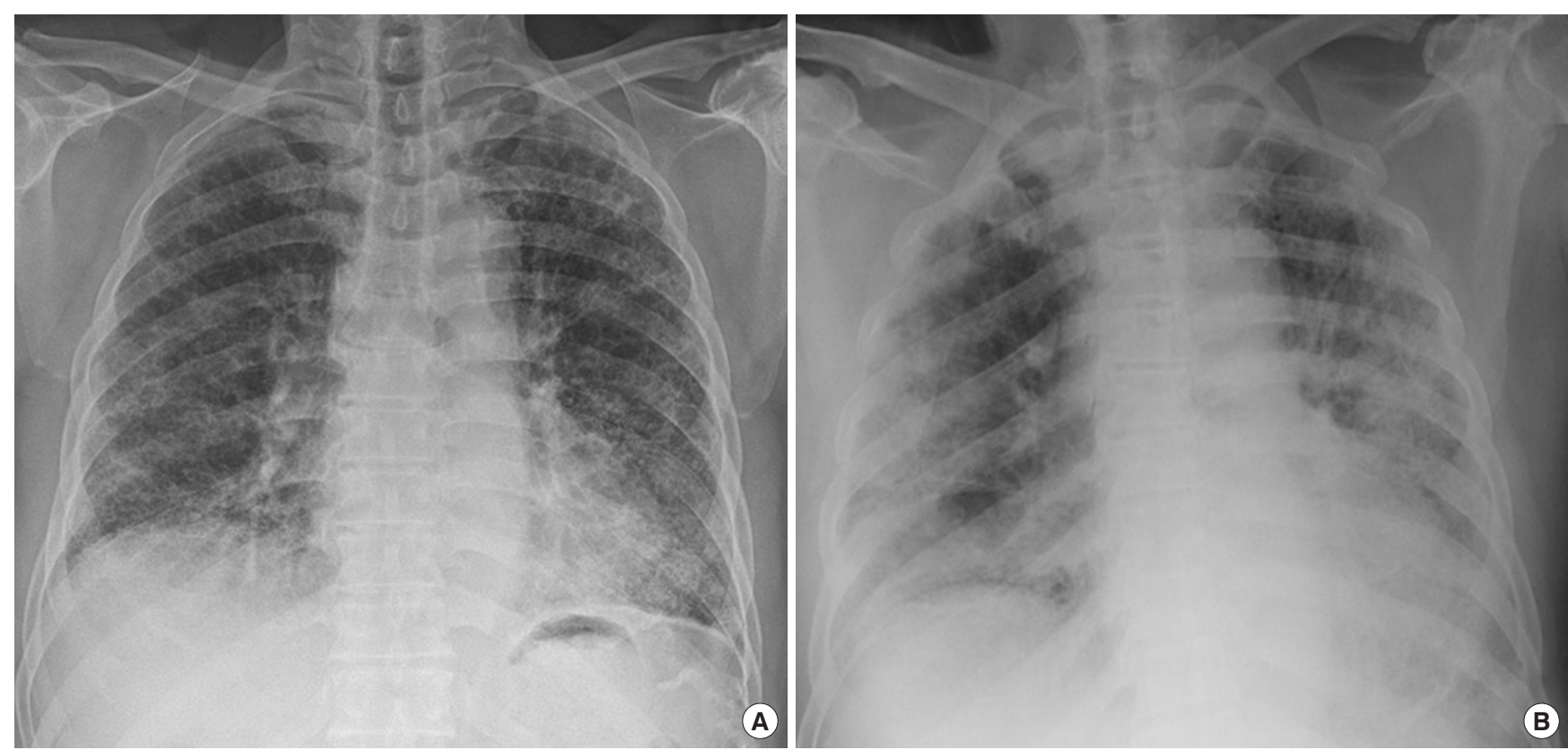

Figure 1. Chest $X$-rays a month before the hospital visit (A) and on emergency room visit (B). (A) Chest X-ray showing reticular opacities in both lower lung fields. (B) Chest X-ray showing an increased infiltration in both lower lung fields. 

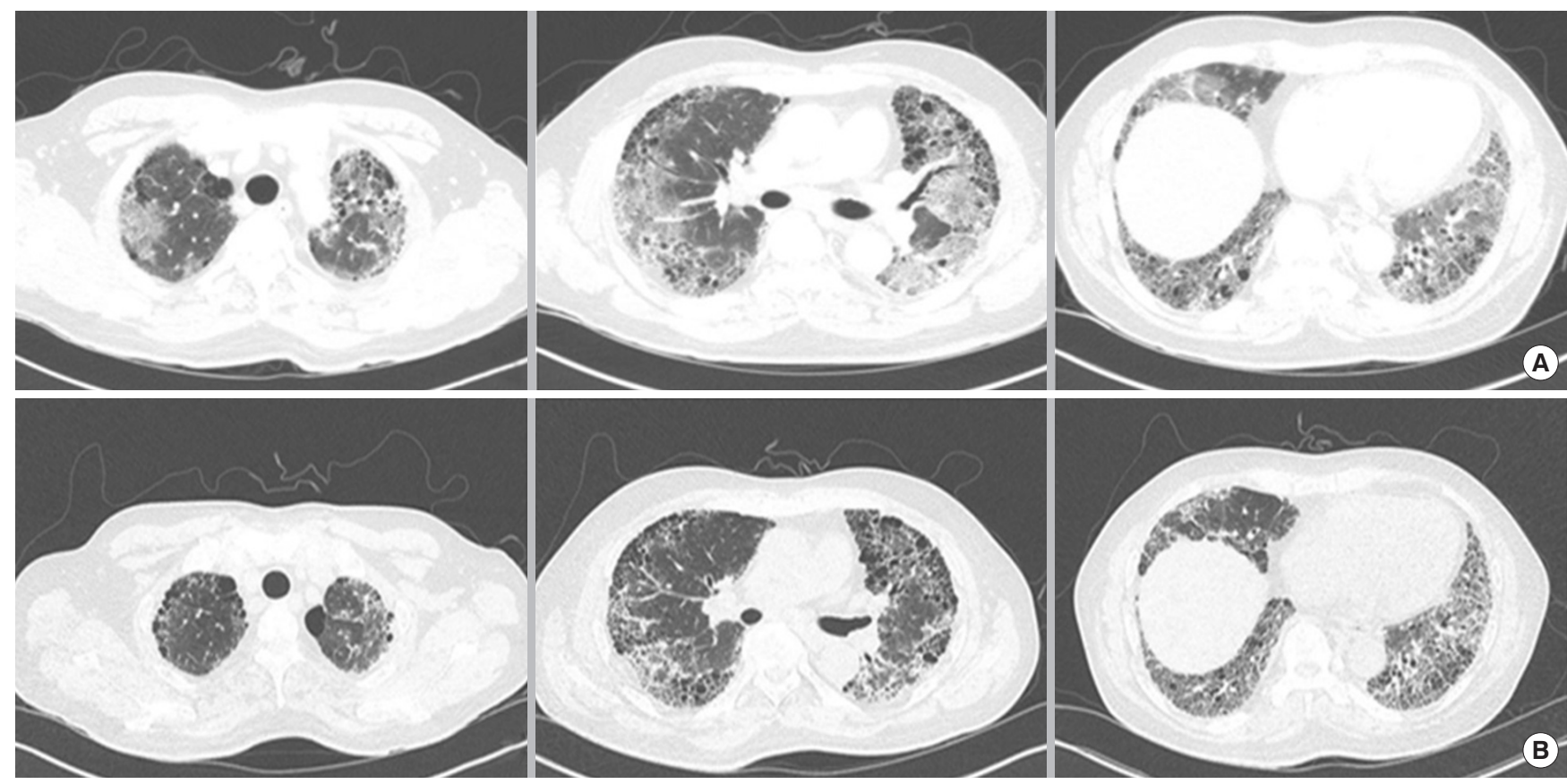

Figure 2. Chest computed tomography findings before (A) and after (B) direct hemoperfusion with a polymyxin B-immobilized fiber column (PMX-DHP) treatment. A chest computed tomography after two cycles of PMX-DHP demonstrates improvements in the ground-glass opacification around underlying honeycombing.

Toray Industries Inc., Tokyo, Japan) at 24-hour intervals for 6 hours each; $\mathrm{PaO}_{2} / \mathrm{FiO}_{2}$ ratio, interleukin-6, and CRP levels were measured at pre- and post-PMX-DHP. The $\mathrm{PaO}_{2} / \mathrm{FiO}_{2}$ ratio improved from 45 to 233 after two cycles of PMX-DHP (Figure 3A). The oxygenation improved and the patient did not require ECMO. IL-6 levels decreased from $79.2 \mathrm{pg} / \mathrm{ml}$ to $5.3 \mathrm{pg} /$ $\mathrm{ml}$ (Figure 3B). CRP levels also decreased from 20.76 to 6.6 mg/dl, 72 hours after PMX-DHP (Figure 3C). After two cycles of PMX-DHP, no redeterioration in blood was noted, as observed by IL-6, CRP, and chest X-rays.

The patient was weaned from mechanical ventilation on day 7 and supplemented with a high flow nasal cannula $\mathrm{FiO}_{2}$ 0.5 and flow of $60 \mathrm{~L} / \mathrm{min}$. After 13 days of hospitalization, the oxygen supply was changed from the high flow nasal cannula $\mathrm{FiO}_{2} 0.4$ and flow $30 \mathrm{~L} / \mathrm{min}$ to a nasal prong $\mathrm{O}_{2} 5 \mathrm{~L} / \mathrm{min}$, and the patient was subsequently transferred to the general ward. Methylprednisolone was tapered gradually after pulse therapy was administered intravenously for 3 days. Administration of pirfenidone, an anti-fibrotic agent, was initiated and was gradually increased to the maximal dose of $1,800 \mathrm{mg} /$ day. Chest CT, 23 days after admission, showed improvements in the groundglass opacity (Figure 2B). Through pulmonary rehabilitation, the patient was able to recover to a state of normal daily life with improved motor skills and was discharged without any neurological damage.

\section{Case 2}

A 73-year-old man presented in the emergency room with dyspnea. The patient was diagnosed with IPF and pirfenidone was administered starting from 12 months ago. The patient suffered AE-IPF and was treated with corticosteroids pulse therapy 4 months ago. The patient was discharged after recovery and then maintained pirfenidone at a dose of $1,800 \mathrm{mg}$ a day. The pulmonary function test indicated that the FEV1/ FVC ratio was $82 \%$, the predicted FVC was $51 \%$, and the predicted DLco predicted was $37 \%$. According to the GAP index, the severity of IPF was estimated as stage 2. Four months after the first-exacerbation, the patient presented in the emergency room with dyspnea in July of 2018. Chest CT showed bilateral ground-glass opacification with reticular shadows. He was diagnosed with AE-IPF for the second time. The $\mathrm{PaO}_{2} / \mathrm{FiO}_{2}$ ratio at that time was 78, with a CRP of $2.73 \mathrm{mg} / \mathrm{dl}$ and procalcitonin of $0.25 \mathrm{ng} / \mathrm{ml}$. The patient's respiration deteriorated and we decided to start mechanical ventilation. At the same time, the patient was treated with corticosteroid pulse therapy, antibiotics and PMX-DHP. The protocol of PMX-DHP was the same as for the case 1 patient. After PMX-DHP, the IL- 6 level, which peaked $133.1 \mathrm{pg} / \mathrm{ml}$ at the end of the first session, soon decreased to $6.2 \mathrm{pg} / \mathrm{ml}$ (Figure 3). The level of IL-6 did not exceed $23.9 \mathrm{pg} / \mathrm{ml}$ after treatment. However, there was no significant change in the $\mathrm{PaO}_{2} / \mathrm{FiO}_{2}$ ratio. The $\mathrm{PaO}_{2} / \mathrm{FiO}_{2}$ ratio 


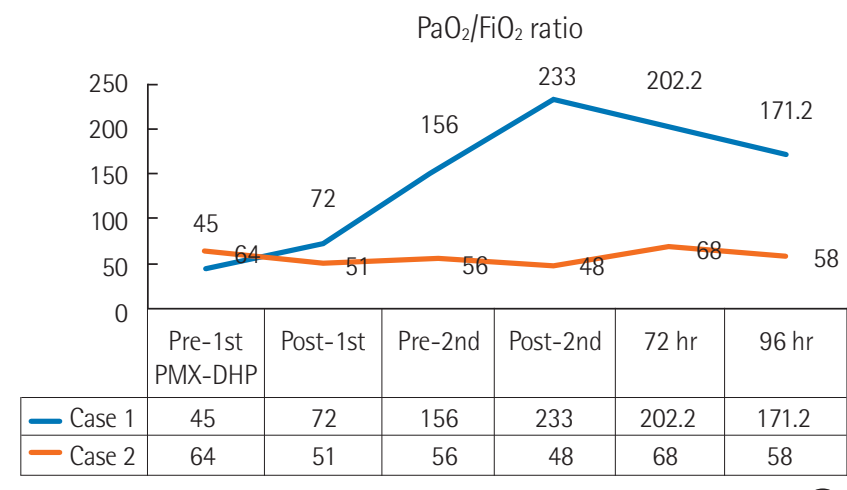

IL-6 (pg/ml)

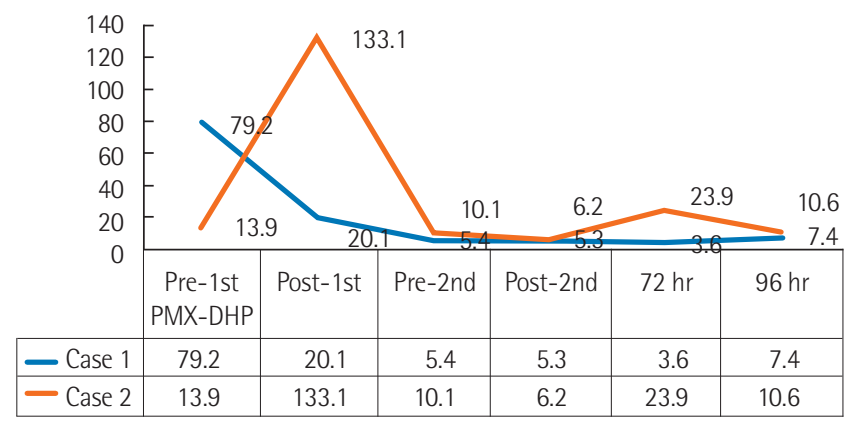

B

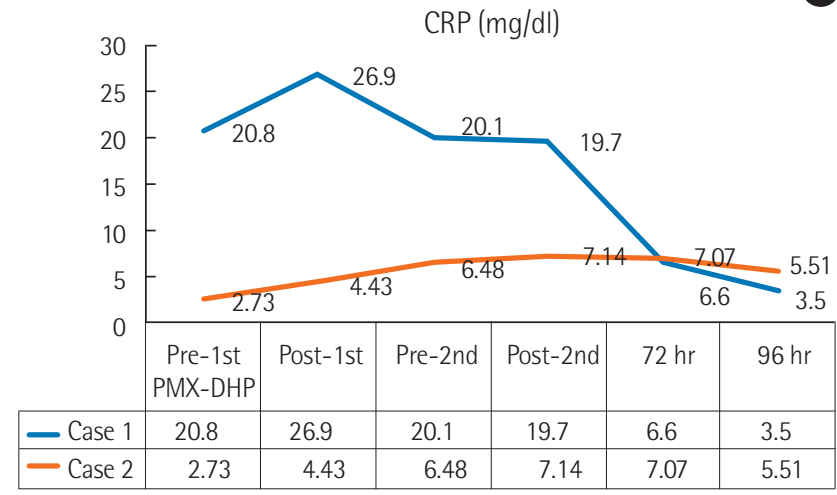

C

Figure 3. The $\mathrm{PaO}_{2} / \mathrm{FiO}_{2}$ ratio (A), interleukin-6 (IL-6) level (B), and C-reactive protein (CRP) level (C) before and after direct hemoperfusion with a polymyxin B-immobilized fiber column (PMX-DHP).

was 64 before treatment, and after two cycles of PMX-DHP treatment it decreased to 48. Ninety-six hours later, the $\mathrm{PaO}_{2} /$ $\mathrm{FiO}_{2}$ ratio was 58 . The patient's respiratory state worsened and he died of respiratory failure on day 8 after admission.

\section{DISCUSSION}

AE-IPF is typically associated with an extremely poor prognosis, with the reported 90-day mortality rate being approximately $60 \%-80 \%$ [8]. Steroids and immune-suppressants have been used to treat AE-IPF, but there are currently no effective treatments [1]. PMX-DHP was developed to remove plasma endotoxins from patients with sepsis. PMX-DHP not only removes plasma endotoxins from Gram-negative bacteria, but may also be effective in patients with Gram-positive bacterial infections and endotoxin negative infections [9]. One study reported that PMX-DHP improved oxygenation and reduced mortality in ARDS patients [10]. In addition to sepsis, the effects on ARDS have been reported in IPF patients. In 2006, the beneficial effects of PMX-DHP were reported in six patients with AE-IPF [11]. Since then, several case reports of AE-IPF patients successfully treated with PMX-DHP have been published. Although the underlying mechanism for the effect of PMX-DHP in AE-IPF is not clear, several studies have shown that PMX-DHP not only reduces various circulating molecules but also activates neutrophils and affects inflammatory cells or mediators. Seo et al. [11] found that IL-6, IL-8, and plasminogen activator inhibitor 1 decreased in all AE-IPF patients that responded to PMX-DHP. Enomoto et al. [2] announced that treatment with PMX-DHP reduced the 12-month mortality in a study on 31 patients with AE-IPF where, the PMX-DHP group showed a significant improvement in the $\mathrm{PaO}_{2} / \mathrm{FiO}_{2}$ ratio, compared with the non-PMX-DHP group, and the PMX-DHP had decreased levels of inflammation and an increased survival rate to more than 12 months ( $48.2 \%$ vs. $5.9 \%, \mathrm{P}=0.041$ ). They also reported that PMX-DHP was relatively safe and was not associated with serious adverse events.

When performing PMX-DHP, similar to continuous renal replacement therapy, regional anticoagulation is indispensable and unfractionated heparin or synthetic serine protease inhibitor nafamostat mesilate (NM) can be used. There are few known side effects of continuous renal replacement therapy and anti-coagulation. In the case of NM, one study has reported few bleeding side effects, along with an anti-inflammatory effect. Therefore, we used an NM of $40 \mathrm{mg} / \mathrm{hr}$ [12]. Data on the effective use time and frequency of PMX-DHP of AEIPF are lacking; additionally, no randomized clinical trials have studied this approach. However, one study reported that PMX-DHP was more effective when applied within 1 week of acute symptom exacerbation onset when the underlying IPF was more severe $[1,2]$. Here, we reported two cases of AE-IPF that were treated by PMX-DHP. Although baseline characteristics and AE-IPF severity were similar, the case 1 patient successfully recovered without ECMO, but the case 2 patient died even after applying PMX-DHP. It is unlikely that PMX-DHP will be effective in all patients with AE-IPF due to multiple clinical factors, and it is also difficult to determine which pa- 
tients should be considered for PMX-DHP. Therefore, additional studies are needed to optimize this therapeutic application. In conclusion, PMX-DHP considerably improved the $\mathrm{PaO}_{2} / \mathrm{FiO}_{2}$ ratio and reduced inflammatory markers (IL-6 and CRP) in a patient with AE-IPF; therefore, PMX-DHP could be helpful for managing patients with AE-IPF. To further determine the effects of PMX-DHP in patients with AE-IPF, a double blind randomized controlled study is needed.

\section{CONFLICT OF INTEREST}

No potential conflict of interest relevant to this article was reported.

\section{ORCID}

Shin Young Kim https://orcid.org/0000-0001-5460-2005

Jin Han Park https://orcid.org/0000-0002-4546-5798

Hyo Jung Kim

Hang Jea Jang

Hyun Kuk Kim

Seung Hoon Kim

Jae Ha Lee

https://orcid.org/0000-0002-8867-7483

https://orcid.org/0000-0001-7733-4365

https://orcid.org/0000-0002-9360-3956

https://orcid.org/0000-0002-0688-2783

https://orcid.org/0000-0003-0932-2826

\section{AUTHOR CONTRIBUTIONS}

Conceptualization: JHL. Data curation: HJK, JHP. Formal analysis: HJJ. Methodology: HKK. Visualization: SHK. Writingoriginal draft: SYK. Writing-review \& editing: SYK, JHL.

\section{REFERENCES}

1. Oishi K, Aoe K, Mimura Y, Murata Y, Sakamoto K, Koutoku W, et al. Survival from an acute exacerbation of idiopathic pulmonary fibrosis with or without direct hemoperfusion with a polymyxin B-immobilized fiber column: a retrospective analysis. Intern Med 2016;55:3551-9.

2. Enomoto N, Mikamo M, Oyama Y, Kono M, Hashimoto D, Fujisawa T, et al. Treatment of acute exacerbation of idiopathic pulmonary fibrosis with direct hemoperfusion using a polymyxin B-immobilized fiber column improves survival. BMC Pulm Med 2015;15:15.
3. Abe S, Azuma A, Mukae H, Ogura T, Taniguchi H, Bando M, et al. Polymyxin B-immobilized fiber column (PMX) treatment for idiopathic pulmonary fibrosis with acute exacerbation: a multicenter retrospective analysis. Intern Med 2012;51: 1487-91

4. Kushi H, Miki T, Nakahara J, Okamoto K, Saito T, Tanjoh K. Hemoperfusion with an immobilized polymyxin B column reduces the blood level of neutrophil elastase. Blood Purif 2006;24:212-7.

5. Komatsu M, Hachiya T, Takahashi H, Yoshikawa S, Honda T. Direct hemoperfusion with polymyxin B-immobilized fiber for the treatment of the acute exacerbation of idiopathic pulmonary fibrosis in patients requiring invasive mechanical ventilation. Respir Investig 2017;55:318-22.

6. Raghu G, Rochwerg B, Zhang Y, Garcia CA, Azuma A, Behr J, et al. An official ATS/ERS/JRS/ALAT clinical practice guideline: treatment of idiopathic pulmonary fibrosis. An update of the 2011 clinical practice guideline. Am J Respir Crit Care Med 2015;192:e3-19.

7. Ley B, Ryerson CJ, Vittinghoff E, Ryu JH, Tomassetti S, Lee JS, et al. A multidimensional index and staging system for idiopathic pulmonary fibrosis. Ann Intern Med 2012;156:684-91.

8. Atsumi K, Saito Y, Kuse N, Kobayashi K, Tanaka T, Kashiwada T, et al. Prognostic factors in the acute exacerbation of idiopathic pulmonary fibrosis: a retrospective single-center study. Intern Med 2018;57:655-61.

9. Tani T, Hanasawa K, Kodama M, Imaizumi H, Yonekawa M, Saito M, et al. Correlation between plasma endotoxin, plasma cytokines, and plasminogen activator inhibitor-1 activities in septic patients. World J Surg 2001;25:660-8.

10. Tsushima K, Kubo K, Koizumi T, Yamamoto H, Fujimoto K, Hora K, et al. Direct hemoperfusion using a polymyxin B immobilized column improves acute respiratory distress syndrome. J Clin Apher 2002;17:97-102.

11. Seo Y, Abe S, Kurahara M, Okada D, Saito Y, Usuki J, et al. Beneficial effect of polymyxin B-immobilized fiber column (PMX) hemoperfusion treatment on acute exacerbation of idiopathic pulmonary fibrosis. Intern Med 2006;45:1033-8.

12. Shimizu T, Miyake T, Kitamura N, Tani M, Endo Y. Endotoxin adsorption: direct hemoperfusion with the polymyxin B-immobilized fiber column (PMX). Transfus Apher Sci 2017;56: 682-8. 